03,08

\title{
Развитие метода локальной симметрии в модели суперъячейки для кристалла с примесью
}

\author{
(C) Р.А. Эварестов, С.И. Лукьянов \\ Санкт-Петербургский государственный университет, Институт химии, \\ Санкт-Петербург, Россия \\ E-mail: s.lukyanov@spbu.ru
}

Поступила в Редакцию 10 декабря 2018 г.

В окончательной редакции 10 декабря 2018 г.

Принята к публикации 11 декабря 2018 г.

\begin{abstract}
Рассмотрены симметрийные аспекты периодической модели кристалла с точечным дефектом (модель суперъячейки или расширенной элементарной ячейки - РЭЯ): расщепление позиций Уайкова в примитивной ячейке кристалла при введении РЭЯ и переклассификация состояний по волновому вектору вследствие соответствующего сужения зоны Бриллюэна. При рассмотрении точечного дефекта в кристалле в модели РЭЯ необходимо учитывать симметрию одноэлектронных состояний исходного кристалла в вершине валентной зоны и на дне зоны проводимости. Выбранная РЭЯ должна воспроизводить эти состояния.

Каждому конкретному выбору РЭЯ соответствует определенное расщепление позиций Уайкова исходного кристалла и как результат - возможность поместить дефект в позиции с разной точечной симметрией, а также провести расчет без учета точечной симметрии кристалла с дефектом вообще (метод сайт-симметрии). По результатам расчета кристалла с дефектом в модели РЭЯ с учетом сайт-симметрии можно определить реальную симметрию кристалла с дефектом, что существенно для интерпретации экспериментальных данных.

Показано, что примесь меди в узле лития в кристалле $\mathrm{LiCl}$ сохраняет кубическую симметрию замещаемого атома, примесь атома железа в узле титана с кубической локальной симметрией в кристалле $\mathrm{SrTiO}_{3}$ понижает симметрию до тетрагональной, а для $H$ центра в кристалле $\mathrm{CsPbI}_{3}$ с образованием иона $\mathrm{I}_{2}^{-}$характерно полное снятие точечной симметрии.
\end{abstract}

DOI: 10.21883/FTT.2019.06.47681.336

\section{1. Введение}

В теории кристаллов с примесными центрами достаточно давно используется модель суперьячейки (supercell, расширенной элементарной ячейки РЭЯ) [1-5]. В этой модели примитивная ячейка исходного кристалла расширяется и в расширенной ячейке примесь помещается либо в междоузлие (межузельный атом), либо вместо атома кристалла-матрицы (примесь замещения), в зависимости от природы рассматриваемого дефекта.

Выбор суперъячейки определяет как симметрию кристалла с периодическим дефектом, так и концентрацию примесных атомов. Популярность такой модели кристалла с примесью определяется возможностью применять для ее реализации современные компъютерные программы, разработанные для неэмпирических расчетов совершенных кристаллов как в базисе ЛКАО (например, [6]), так и в базисе плоских волн (например, [7]).

При введении расширенной ячейки для исходного кристалла позиции Уайкова для его пространственной группы расщепляются. Это расщепление можно установить, используя программу WYCKSPLIT на сервеpe [8] До последнего времени это обстоятельство не принималось во внимание: замещаемый примесью атом помещался в начало координат и кристалл с примесью в модели расширенной ячейки обладал точечной сим- метрией локальной группы замещаемого атома. Учет указанного расщепления может привести к понижению рассчитанной энергии образования дефекта и поэтому существен при описании кристалла с примесью. Этот вывод подтверждается конкретными расчетами энергии образования центров разной природы: $\mathrm{ZnO}: \mathrm{C}$ [9], междоузельных атомов кислорода в кристаллах корунда [10] и шпинели [11], вакансий в кристаллах $\mathrm{CeO}_{2}$ [12] и $\mathrm{ZnO}[5]$.

В настоящей работе описывается дальнейшее развитие метода учета локальной симметрии в модели расширенной ячейки для кристалла с дефектом. Предлагается проводить расчет примесного центра (как замещения, так и в междоузлии) с оптимизацией геометрии как при точечной симметрии расщепленных позиций замещаемого атома или междоузлия, так и с полным снятием указанной симметрии. Сравнение полученных энергий для всех рассмотренных типов симметрии кристалла с дефектом позволяет определить, какая из них соответствует реальному дефекту.

Важной характеристикой кристалла с точечным дефектом является расположение примесных уровней в запрещенной зоне исходного кристалла. Поэтому при выборе РЭЯ следует учитывать симметрию одноэлектронных состояний в вершине валентной зоны и на дне зоны проводимости в исходном кристалле. Рассмотренные в настоящей работе кристаллы отличаются по 
симметрии состояний на границах запрещенной зоны: в кристалле $\mathrm{LiCl}$ щель прямая (в точке Г), в кристалле $\mathrm{SrTiO}_{3}$ вершине валентной зоны соответствует точка $R$, а дну зоны проводимости - точка $\Gamma$ (непрямая щель), a в кристалле $\mathrm{CsPbI}_{3}$ щель прямая в точке $R$. При введении РЭЯ изменяется классификация одноэлектронных состояний исходного кристалла по волновому вектору. Это необходимо учитывать в расчете кристалла с дефектом.

Статья организована следующим образом. В разделе 2 кратко описывается метод расширенной ячейки и его применение для кубических кристаллов, рассмотренных в настоящей работе.

В разделе 3 обсуждается расщепление позиций Уайкова при расширении элементарной ячейки и учет этого расщепления в методе сайт-симметрии для кристаллов с дефектами.

В разделе 4 предлагаемый подход применяется к примесному атому меди в кристалле LiCl. Показано, что в этом случае энергия суперьячейки кристалла с примесью практически не изменяется при оптимизации геометрии в расчете с максимально возможной (кубической) симметрией и без учета симметрии вообще.

В разделе 5 предлагаемый подход применяется к примесному центру $\mathrm{SrTiO}_{3}: \mathrm{Fe}$, многократно рассчитанному ранее на основе традиционного подхода то есть при помещении примеси железа вместо атома титана в позицию с кубической симметрией. Показано, что развиваемый в настоящей работе подход предсказывает не кубическую, а тетрагональную симметрию такого центра, в согласии с имеющимися экспериментальными данными.

В разделе 6 рассмотрен междоузельный атом йода (H-центр) в кристалле $\mathrm{CsPb}_{3}$ (широко изучаемом в настоящее время материале для создания солнечных батарей). Показано, что образование дефекта молекулярного типа $\mathrm{I}_{2}^{-}$можно подтвердить теоретически только при полном снятии локальной симметрии.

Работа завершается изложением основных ее результатов.

\section{2. Модель суперьячейки для кристалла с примесью}

Рассмотрим кратко преобразование расширенная ячейка-суженная зона Бриллюэна (supercell-zone-folding transformation). Подробное рассмотрение этого преобразования выполнено в [4].

Пусть заданы вектора трансляции примитивной ячейки исходного кристалла $\mathbf{a}_{1}, \mathbf{a}_{2}, \mathbf{a}_{3}$ и соответствующие им вектора трансляции обратной решетки $\mathbf{B}_{1}$, $\mathbf{B}_{2}, \mathbf{B}_{3}$, определяющие зону Бриллюэна (ЗБ), то есть ячейку Вигнера-Зейтца обратной решетки. При этом $\mathbf{B}_{i} \cdot \mathbf{a}_{j}=2 \pi \delta_{i j}, i, j=1,2,3$. Волновые вектора $\mathbf{k}$ из зоны Бриллюэна - это индексы неприводимых представлений группы трансляций $T$, заданной векторами $\mathbf{a}_{1}, \mathbf{a}_{2}, \mathbf{a}_{3}$.
Суперячейка исходной прямой решетки (расширенная ячейка) определяется векторами трансляции $\mathbf{A}_{j}^{L}$ новой прямой решетки

$$
\mathbf{A}_{j}^{L}=\sum_{i=1}^{3} L_{j i} \mathbf{a}_{i}, \quad j=1,2,3,
$$

где целочисленная матрица $L$ связывает вектора трансляции новой и исходной прямых решеток. Определитель этой матрицы $N=\operatorname{det}(L)$ равен числу примитивных ячеек в расширенной ячейке. По определению, преобразование (1) является симметричным, если оно сохраняет точечную группу симметрии исходной решетки. При этом тип исходной решетки может измениться (в настоящей работе рассмотрена модель РЭЯ для преобразований (1), сохраняющих кубическую точечную симметрию решетки). В модели РЭЯ вместо группы симметрии исходного кристалла $G=T F$ (группа кристаллического класса $F$ изоморфна фактор-группе $G / T$ ) рассматривается ее подгруппа $G^{L}=T^{L} F^{L}$.

Заметим, что сохранение точечной группы симметрии $F$ прямой решетки обеспечивает и сохранение точечной группы симметрии исходного кристалла (кристаллического класса), которая либо совпадает с точечной группой симметрии решетки или является ее подгруппой. Процедура поиска симметричных преобразований РЭЯ описана в [13], где матрицы таких преобразований приведены для всех 14 решеток Браве.

Преобразование (1) векторов трансляции прямой решетки приводит к преобразованию всего прямого пространства

$$
\mathbf{r}_{i}^{L}\left(\mathbf{r}_{0}^{L}\right)=\mathbf{r}_{0}^{L}+\sum_{j=1}^{3} m_{j i} \mathbf{a}_{j}^{L}, \quad i=1,2, \ldots, N
$$

где $\mathbf{r}_{i}^{L}$ и $\mathbf{r}_{0}^{L}-$ координаты точек прямого пространства после преобразования, причем точка $\mathbf{r}_{0}^{L}$ находится внутри РЭЯ, а $m_{j i}$ - целые числа или ноль. Иными словами, в правой части соотношения (2) оказываются координаты всех точек прямого пространства, расположенных внутри РЭЯ, в том числе и расщепленных за счет перехода к РЭЯ позиций Уайкова исходной пространственной группы.

В настоящей работе рассматриваются примесный центр $\mathrm{LiCl}: \mathrm{Cu}$ (исходный кристалл $\mathrm{LiCl}$ имеет симметрию группы $F m \overline{3} m$ с гранецентрированной кубической решеткой) и примесные центры $\mathrm{SrTiO}_{3}: \mathrm{Fe}$, $\mathrm{CsPbI}_{3}$ : I (исходные кристаллы $\mathrm{SrTiO}_{3}$ и $\mathrm{CsPbI}_{3}$ со структурой перовскита имеют симметрию группы $P m \overline{3} m$ с простой кубической решеткой). Матрицы симметричных преобразований, сохраняющие тип кубической решетки $L(P \rightarrow P)=L(F \rightarrow F)$ или изменяющие его $L(F \rightarrow P)=L(P \rightarrow I), L(P \rightarrow F), L(F \rightarrow I)$ имеют вид [13]:

$$
L(P \rightarrow P)=L(F \rightarrow F)=\left(\begin{array}{lll}
n & 0 & 0 \\
0 & n & 0 \\
0 & 0 & n
\end{array}\right), \quad N=n^{3}
$$




$$
\begin{gathered}
L(P \rightarrow F)=\left(\begin{array}{lll}
0 & n & n \\
n & 0 & n \\
n & n & 0
\end{array}\right), \quad N=2 n^{3}, \quad \text { (3b) } \\
L(F \rightarrow P)=L(P \rightarrow I)=\left(\begin{array}{ccc}
-n & n & n \\
n & -n & n \\
n & n & -n
\end{array}\right), \quad N=4 n^{3}, \\
L(F \rightarrow I)=\left(\begin{array}{ccc}
3 n & -n & -n \\
-n & 3 n & -n \\
-n & -n & 3 n
\end{array}\right), \quad N=16 n^{3} .
\end{gathered}
$$

В (3) $N=\operatorname{det}(L)-$ число примитивных ячеек в расширенной ячейке, $n$ - целые числа.

В качестве примера рассмотрим преобразование (3d) для позиций Уайкова $a(0,0,0), \quad b(0.5,0.5,0.5)$, $2 c(0.25,0.25,0.25), 6 d(0,0.25,0.25)$ пространственной группы симметрии $F m \overline{3} m$ кристалла $\mathrm{LiCl}$ (координаты в скобках заданы в векторах трансляции ячейки гране-центрированной кубической (гцк) решетки). Преобразование (3d) при $n=1$ увеличивает примитивную ячейку гцк-решетки в 16 раз и построенная из РЭЯ решетка является объемно-центрированной кубической (оцк). Подгруппа трансляций $T^{L}$ для решетки из РЭЯ имеет индекс 16 относительно исходной группы трансляций $T$. Координаты позиций Уайкова для исходной пространственной группы $F m \overline{3} m$ и для ее подгруппы $\operatorname{Im} \overline{3} m$ связаны соотношениями

$$
\begin{gathered}
a=a^{(16)}(0,0,0)+3 b^{(16)}(0,0.5,0.5) \\
+12 h^{(16)}(0.25,0.25,0) \\
b=4 c^{(16)}(0.25,0.25,0.25)+6 e^{(16)}(0.25,0,0) \\
+6 d^{(16)}(0.25,0,0.5) \\
2 c=8 f^{(16)}(0.125,0.125,0.125) \\
+24 k^{(16)}(0.125,0.125,0.625) \\
6 d=12 h^{(16)}(0,0.125,0.125)+12 h^{(16)}(0,0.375,0.375) \\
+24 i^{(16)}(0.25,0.125,0.375)+24 j^{(16)}(0,0.125,0.625) \\
+24 k^{(16)}(0.25,0.125,0.875)
\end{gathered}
$$

Соотношения (4) написаны для кристаллографических (кубических) ячеек гцк- и оцк-решеток и связывает координаты находящихся внутри РЭЯ позиций Уайкова исходной группы $G=F m \overline{3} m$ (с подгруппой трансляций $T$ ) и ее подгруппы $G^{L}=(\operatorname{Im} \overline{3} m)^{L}$ (с подгруппой трансляций $\left.T^{L}(L=16)\right)$. Соотношения (4) написаны для симметричного преобразования от гцк- К оцк-решетке и поэтому выполняются для целых орбит (позиций Уайкова) в прямой решетке.

Из соотношений (4) следует, что при переходе к РЭЯ происходит расщепление исходных позиций Уайкова. Среди расщепленных позиций появляются таковые с точечной симметрией, пониженной по сравнению с точечной симметрией исходной позиции. В частности, из соотношения (4a) видно, что при расщеплении позиции $a\left(\begin{array}{lll}0 & 0 & 0\end{array}\right)$ с кубической локальной симметрией появляются позиции $3 b$ и $12 h$ с более низкой локальной симметрией $D_{4 h}$ и $C_{2 v}$ соответственно. Поэтому расчет примеси, замещающей атом исходного кристалла, следует проводить с учетом расщепления позиций Уайкова при введении РЭЯ, как это и делается в методе сайтсимметрии для кристаллов с дефектами (см. следующий раздел).

Соотношения (4) получены с помощью программ CELLSUB и WYCKSPLIT, доступных на сервере BCS [8], где расщепление позиций Уайкова при переходе от пространственной группы к ее подгруппе можно определить, задав индекс подгруппы в группе и матрицу преобразования от подгруппы к группе. Подробное описание этой процедуры дано в [5].

При расширении ячейки в прямой решетке происходит сужение зоны Бриллюэна (zone-folding) в обратной решетке. Вектора трансляции новой обратной решетки $\mathbf{b}^{L}=\left(L^{T}\right)^{-1} \mathbf{B}$, a $(N-1)$ точек $\mathbf{k}_{i}^{L}$ исходной зоны Бриллюэна становятся эквивалентны точке $\mathbf{k}_{0}^{L}$ суженной зоны Бриллюэна (СЗБ)

$$
{ }^{*} \mathbf{k}_{i}^{L}\left(\mathbf{k}_{0}^{L}\right)={ }^{*} \mathbf{k}_{0}^{L}+\sum_{j=1}^{3} m_{j i} \mathbf{b}_{j}^{L} \quad i=1,2, \ldots, N .
$$

В (5) $m_{j i}$ - целые числа или ноль. Для симметричного преобразования в прямой решетке к звезде точки ${ }^{*} \mathbf{k}_{0}^{L}$ приводятся целые звезды векторов исходной зоны Бриллюэна. Соотношение (5) связывает индексы $\mathbf{k}^{L}$ неприводимых представлений подгруппы $T^{L} \mathrm{c}$ индексами $\mathbf{k}$ неприводимых представлений группы $T$ и может рассматриваться как результат индуцирования представлений группы $T$ с представлений подгруппы $T^{L}$.

Соотношения (1), (5) используются и в модели циклического кластера (квазимолекулярной расширенной элементарной ячейки) $[14,15]$. В отличие от модели РЭЯ-СЗБ, в модели циклического кластера рассматривается квазимолекула - молекулярный кластер в форме РЭЯ с наложением циклических граничных условий на одноэлектронные функции. Остальной кристалл при этом учитывается в виде внешнего поля, а в суженной зоне Бриллюэна рассматривается только точка $\mathbf{k}^{L}=0$. Модель циклического кластера описывает одиночный дефект в кристалле, а модель РЭЯ-СЗБ - периодический дефект. Поэтому в модели РЭЯ-СЗБ необходимо рассматривать состояния кристалла с дефектом в различных точках суженной зоны Бриллюэна, используя соотношение (5). При достаточно большом периоде дефекта обе модели становятся эквивалентны так как дисперсия зоны примесных состояний становится малой.

В табл. 1 и 2 приведены соотношения (5) между точками симметрии исходной и суженной зон Бриллюэна для значений $n=1$, 2. Координаты точек $\mathbf{k}$ исходной ЗБ заданы в векторах трансляции исходной обратной 
Таблица 1. Преобразования РЭЯ-СЗБ для простой кубической решетки $P$

\begin{tabular}{c|c|c|c}
\hline Точки симметии $\mathbf{k}$ & $L(P \rightarrow P)=\left(\begin{array}{lll}2 & 0 & 0 \\
0 & 2 & 0 \\
0 & 0 & 2\end{array}\right)$ & $L(P \rightarrow F)=\left(\begin{array}{lll}0 & 1 & 1 \\
1 & 0 & 1 \\
1 & 1 & 0\end{array}\right)$ & $L(P \rightarrow I)=\left(\begin{array}{cc}-1 & 1 \\
1 & 1 \\
-1 & 1 \\
1 & -1\end{array}\right)$ \\
в исходной ЗБ & $n=2, N=8$ & $n=1, N=2$ & $n=1, N=4$ \\
\hline$\Gamma(0,0,0)$ & $\Gamma^{\prime}: \Gamma, 3 X, 3 M, R$ & $\Gamma^{\prime}: \Gamma, R$ & $\Gamma^{\prime}: \Gamma, 3 M$ \\
\hline $3 X(1 / 2,0,0)$ & $3 X^{\prime}(1 / 2,0,0): 6(1 / 4,0,0)$ & $3 X^{\prime}(1 / 2,0,1 / 2):$ & $H^{\prime}(1 / 2,1 / 2,1 / 2):$ \\
& $12(1 / 2,-1 / 4,0), 6(1 / 2,1 / 2,1 / 4)$ & $3 X, 3 M$ & $2 P^{\prime}(1 / 4,1 / 4,1 / 4):$ \\
\hline $3 M(1 / 2,1 / 2,0)$ & $3 M^{\prime}(1 / 2,1 / 2,0): 12(1 / 4,1 / 4,0)$ & $4 L^{\prime}(1 / 2,1 / 2,1 / 2):$ & $8(1 / 4,1 / 4,1 / 4)$ \\
\hline \multirow{2}{*}{$R(1 / 2,1 / 2,1 / 2)$} & $12(1 / 2,1 / 4,-1 / 4)$ & $8(1 / 4,1 / 4,1 / 4)$ & $6 N^{\prime}(0,0,1 / 2): 12(1 / 4,1 / 4,0)$ \\
& $R^{\prime}(1 / 2,1 / 2,1 / 2):$ & $6 W^{\prime}(1 / 2,1 / 4,3 / 4):$ & $12(1 / 4,1 / 2,1 / 4)$
\end{tabular}

Таблица 2. Преобразования РЭЯ-СЗБ для гранецентрированной кубической решетки $F$

\begin{tabular}{|c|c|c|c|}
\hline $\begin{array}{c}\text { Точки симметрии } \mathbf{k} \\
\text { в исходной ЗБ }\end{array}$ & $\begin{array}{c}L(F \rightarrow F)=\left(\begin{array}{lll}2 & 0 & 0 \\
0 & 2 & 0 \\
0 & 0 & 2\end{array}\right) \\
n=2, N=8\end{array}$ & $\begin{array}{c}L(F \rightarrow P)=\left(\begin{array}{ccc}-1 & 1 & 1 \\
1 & -1 & 1 \\
1 & 1 & -1\end{array}\right) \\
n=1, N=4\end{array}$ & $\begin{array}{c}L(F \rightarrow I)=\left(\begin{array}{ccc}3 & -1 & -1 \\
-1 & 3 & -1 \\
-1 & -1 & 3\end{array}\right) \\
n=1, N=16\end{array}$ \\
\hline$\Gamma(0,0,0)$ & $\Gamma^{\prime}: \Gamma, 3 X, 4 L$ & $\Gamma^{\prime}: \Gamma, 3 X$ & $\Gamma^{\prime}: \Gamma, 3 X, 12(1 / 2,1 / 4,1 / 4)$ \\
\hline $3 X(1 / 2,0,1 / 2)$ & $\begin{array}{c}3 X^{\prime}(1 / 2,0,1 / 2): 6(1 / 4,0,1 / 4) \\
12(-1 / 4,0,1 / 4), 6(1 / 4,1 / 2,-1 / 4)\end{array}$ & $\begin{array}{l}3 X^{\prime}(1 / 2,0,0): 6 W \\
\quad 6(1 / 4,0,1 / 4)\end{array}$ & $\begin{array}{c}H^{\prime}(1 / 2,1 / 2,1 / 2): 4 L, 6 W \\
6(1 / 4,1 / 4,0)\end{array}$ \\
\hline $4 L(1 / 2,1 / 2,1 / 2)$ & $\begin{array}{c}4 L^{\prime}(1 / 2,1 / 2,1 / 2): 8(1 / 4,1 / 4,1 / 4) \\
24(-1 / 4,1 / 4,1 / 4)\end{array}$ & $3 M^{\prime}(1 / 2,1 / 2,0): 12(1 / 4,1 / 4,1 / 2)$ & $\begin{array}{c}6 N^{\prime}(0,0,1 / 2): 12(1 / 8,1 / 8,1 / 4) \\
12(-1 / 4,3 / 8,3 / 8), 24(3 / 8,-3 / 8,1 / 2) \\
24(-1 / 2,1 / 8,3 / 8), 24 v(3 / 8,1 / 4,3 / 8)\end{array}$ \\
\hline $6 W(1 / 2,1 / 4,3 / 4)$ & $\begin{array}{c}6 W^{\prime}(1 / 2,1 / 4,3 / 4): \\
24(-1 / 4,1 / 8,3 / 8), 24(1 / 4,1 / 8,3 / 8)\end{array}$ & $R^{\prime}(1 / 2,1 / 2,1 / 2): 4 L$ & $\begin{array}{c}2 P^{\prime}(1 / 4,1 / 4,1 / 4): 8(1 / 4,1 / 4,1 / 4) \\
24(1 / 4,1 / 2,1 / 2)\end{array}$ \\
\hline
\end{tabular}

решетки, штрихом отмечены точки симметрии суженной ЗБ, заданные в векторах трансляции новой обратной решетки. Эти результаты можно получить как с помощью свободно распространяемой программы И.И. Тупицина genkpt [4], так и с учетом связи между координатами точек в прямой и обратной решетках, представленной на сервере bcs [8]. В рассмотренном выше примере к каждой точке суженной зоны Бриллюэна объемноцентрированной кубической решетки приводятся 15 точек исходной зоны Бриллюэна гранецентрированной кубической решетки. Используя соотношения (4) для точек прямой решетки и соответствие точек прямой и обратной решеток, приведенное на сервере BCS [8], можно получить приведенное в табл. 2 соответствие точек суженной и исходной зон Бриллюэна для перехода от гранецентрированной кубической решетки к объемноцентрированной кубической решетке

$$
\Gamma^{\prime}: \Gamma+3 X+12(1 / 2,1 / 4,1 / 4)
$$

$$
H^{\prime}(1 / 2,1 / 2,1 / 2): 4 L+6 W+6(1 / 4,1 / 4,0)
$$

$2 P^{\prime}(1 / 4,1 / 4,1 / 4): 8(1 / 4,1 / 4,1 /, 4)+24(1 / 4,1 / 2,1 / 2)$;

$$
\begin{gathered}
6 N^{\prime}(0,0,1 / 2): 12(1 / 8,1 / 8,1 / 4)+12(-1 / 4,3 / 8,3 / 8) \\
+24(3 / 8,-3 / 8,1 / 2)+24(-1 / 2,1 / 8,3 / 8) \\
+24(3 / 8,1 / 4,3 / 8)
\end{gathered}
$$

Заметим, что в (6) точки симметрии со штрихом заданы в векторах трансляции суженной зоны Бриллюэна, а приводящиеся к ним точки исходной зоны Бриллюэна в векторах трансляции исходной обратной решетки.

\section{3. Метод сайт-симметрии для кристаллов с дефектами. Выбор схемы расчета}

Как отмечалось во введении, при традиционном подходе к рассмотрению кристалла с примесью в модели РЭЯ замещаемый в исходном кристалле атом помещают в начало координат и поэтому кристалл с дефектом имеет максимально возможную точечную симметрию, то есть симметрию локальной группы позиции Уайкова, занятой замещаемым атомом. При таком подходе, в частности, примесный атом меди в кристалле $\mathrm{LiCl}$ и примесный атом железа в кристалле $\mathrm{SrTiO}_{3}$ помещают 
в позицию с кубической локальной симметрией, так как замещаемые атомы лития и титана в исходных кристаллах занимают позицию Уайкова $a(0,0,0)$ с кубической локальной симметрией. С увеличением размера суперъячейки кубическая симметрия кристалла с дефектом при таком (традиционном) подходе сохраняется, как это было в расчетах [16] примеси железа в кристалле $\mathrm{SrTiO}_{3}$. При этом в [16] делается неверный вывод, что расположение примеси в РЭЯ не влияет на результаты расчета энергии образования дефекта.

Однако при введении РЭЯ для исходного кристалла занятые атомами позиции расщепляются (см. соотношение (4) для пространственной группы $F m \overline{3} m$ ) и это необходимо учитывать при моделировании кристалла с примесью. Так, в работе [17] рассмотрен кристалл $\mathrm{ZnO}$ с примесью кобальта в узле цинка и показано, что при увеличении ячейки исходного кристалла со структурой вюртцита возникают различные конфигурации магнитных атомов, в зависимости от выбора замещаемой позиции атома цинка в суперъячейке.

Использование программ, имеющихся на кристаллографическом сервере [8], позволяет проанализировать изменение расщепления позиций Уайкова при различном выборе суперьячейки исходного кристалла. При увеличении суперъячейки расщепленные позиции могут обладать различной локальной симметрией и это должно учитываться при помещении примеси в суперъячейку. Подробное рассмотрение этой проблемы проведено в [5] как для симморфных $(P m \overline{3} m, F m \overline{3} m)$, так и для несимморфной $P 6_{3} m c$ пространственных групп. Описываемый подход известен как метод сайт симметрии в модели суперъячейки для кристаллов с дефектами и успешно применен для описания различных дефектов в кристаллах: вакансии кислорода в кристалле $\mathrm{CeO}_{2}$ [12], примеси углерода в кристалле $\mathrm{ZnO}$ [9], вакансий металла и кислорода в кристалле $\mathrm{ZnO}$ [5], междоузельных атомов кислорода в кристаллах корунда $\mathrm{Al}_{2} \mathrm{O}_{3}$ [10] и шпинели $\mathrm{MgAl}_{2} \mathrm{O}_{4}$ [11].

Из результатов всех упомянутых работ следует, что рассчитанная энергия образования дефекта в модели суперьячейки зависит от локальной симметрии позиции Уайкова, в которую помещается дефект. При понижении локальной симметрии оптимизация геометрии кристалла с дефектом приводит к понижению энергии благодаря увеличению числа степеней свободы для каждого атома в РЭЯ. Казалось бы, полное снятие симметрии кристалла с дефектом, приводящее к трем степеням свободы при оптимизации геометрии, должно всегда приводить к максимальному понижению энергии. Но в действительности это зависит от исходного кристалла и природы примеси, что позволяет путем расчета предсказать симметрию дефекта в кристалле и использовать ее при интерпретации экспериментальных данных для электронных и фононных состояний дефектного кристалла.

В настоящей работе проводится дальнейшее развитие метода сайт симметрии для модели РЭЯ. На примере центра $\mathrm{LiCl}: \mathrm{Cu}$ показано, что полное снятие кубической симметрии замещаемого атома Li не приводит к существенному понижению энергии образования дефекта и это доказывает, что такой центр имеет кубическую симметрию.

Для примесного центра $\mathrm{SrTiO}_{3}$ : Fe ситуация оказывается иной. Показано, что тетрагональная симметрия кристалла с дефектом приводит к полной энергии, которая практически не изменяется при дальнейшем понижении симметрии.

Наконец, для междоузельного атома йода (H-центр) в кристалле $\mathrm{CsPbI}_{3}$ только расчет с полным снятием локальной симметрии (три свободных параметра при оптимизации геометрии) приводит к результату, качественно согласующемуся с данными эксперимента о возможности образования иона $\mathrm{I}_{2}^{-}$.

Обсуждаемые ниже расчеты проведены в базисе ЛКАО по программе CRYSTAL17 [6] с использованием гибридных обменно-корреляционных функционалов плотности PBE0 [18] (для кристаллов $\mathrm{LiCl}$ и $\mathrm{SrTiO}_{3}$ и примесей в них) и PBESOL0 [19] (для кристалла $\mathrm{CsPbI}_{3}$ и примеси йода в нем). Для атомов $\mathrm{Li}, \mathrm{Cl}$ и $\mathrm{Cu}$ использован полноэлектронный базис из [20], адаптированный к расчету кристаллических систем. Для кристалла $\mathrm{SrTiO}_{3}: \mathrm{Fe}$ использован базис из [21], с оптимизированными внешними экспонентами гауссовых функций и включающий поляризующие d-функции для атома Sr.

В расчетах кристалла $\mathrm{CsPbI}_{3}$ использованы атомные базисные функции с сайта программы CRYSTAL [20], в которых взаимодействие остова с валентными электронами описывается с помощью эффективного потенциала остова.

Суммирование по зоне Бриллюэна проводилось по методу Монкхорста-Пака [22], с использованием сети $\mathbf{k - т о ч е к ~} 8 \times 8 \times 8$. Точность самосогласования при расчете электронной энергии выбрана $10^{-8} \mathrm{eV}$, все расчеты проведены с полной оптимизацией геометрии рассматриваемых систем.

\section{4. Примесь с сохранением локальной симметрии замещаемого атома: $\mathrm{LiCl}: \mathbf{C u}$}

В табл. 3 приведены результаты DFT PBE0 расчета кристаллов $\mathrm{LiCl}$ и $\mathrm{CuCl}$ с симметрией пространственной группы $F m \overline{3} m$ и оптимизацией постоянной для гцкрешетки. Из табл. 3 видно, что рассчитанные значения постоянной решетки $a$, ширины запрещенной зоны $E_{g}$ и ширины валентной зоны $E_{v}$ (для кристалла $\mathrm{LiCl}$ ) хорошо согласуются с данными эксперимента. Рассчитанные заряды на атомах подтверждают известный факт, что эти кристаллы являются ионными.

На рис. 1, a приведена зонная структура кристалла $\mathrm{LiCl}$. Этот кристалл является изолятором с шириной запрещенной зоны $9.4 \mathrm{eV}$, щель прямая (в точке Г зоны Бриллюэна), верхняя валентная зона образована 

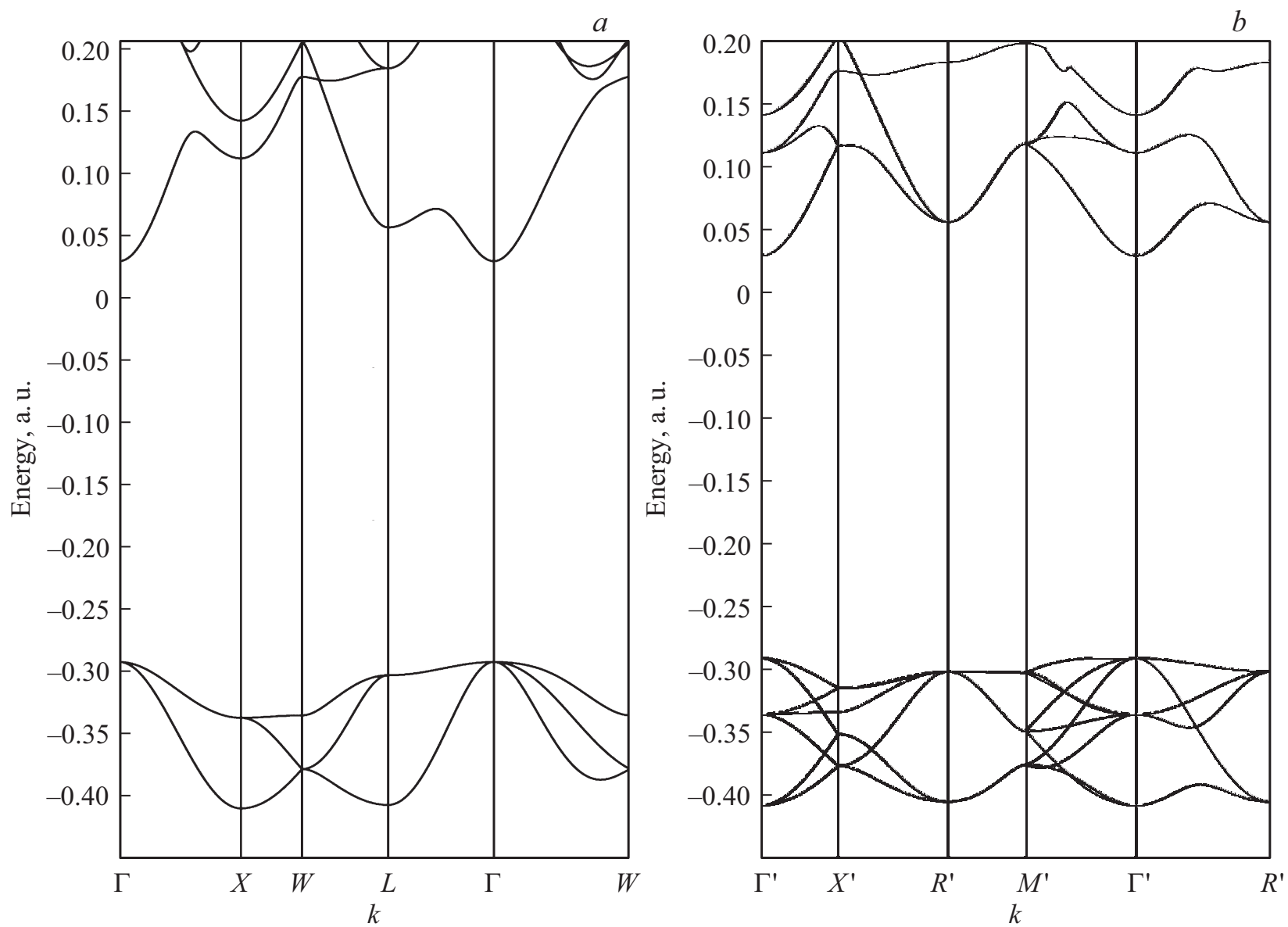

Pис. 1. Зонная структура кристалла LiCl: $a$-расчет с примитивной ячейкой; $b-$ расчет с учетверенной ячейкой.

$3 p$-состояниями хлора, нижняя зона проводимости $2 s$-состояниями лития, см. табл. 3.

На рис. 1, $b$ приведена зонная структура этого кристалла при расчете с учетверенной ячейкой и соответствующей классификацией состояний по волновому вектору в зоне Бриллюэна простой кубической решетки. Из рис. $1, b$ видно, что симметрия состояний на границах запрещенной зоны не изменяется, что существенно для расчета дефекта.

Таблица 3. Свойства кристаллов $\mathrm{LiCl}$ и $\mathrm{CuCl} . a(\AA), E_{g}(\mathrm{eV})$, $E_{v}(\mathrm{eV}), R(\AA)-$ постоянная решетки, ширина запрещенной и верхней валентной зон, расстояние между ближайшими атомами соответственно

\begin{tabular}{c|c|c}
\hline Свойство & $\mathrm{LiCl}$ & $\mathrm{CuCl}$ \\
\hline$a$ (эксп.) & 5.12 & 5.14 \\
$a$ (расч.) & 5.08 & 4.92 \\
$E_{g}$ (экспер.) & 9.4 & 3.2 \\
$E_{g}$ (расч.) & 8.7 & 3.4 \\
$R_{\mathrm{Me}-\mathrm{Cl}}$ & 2.558 & 2.568 \\
$|\mathbf{q}|$ & 0.95 & 0.91 \\
$E_{v}$ (экспер.) & 3.6 & - \\
$E_{v}$ (расч.) & 3.2 & 2.0
\end{tabular}

Кристалл $\mathrm{CuCl}$ - полупроводник с шириной запрещенной зоны $3.2 \mathrm{eV}$, верхняя валентная зона в этом кристалле более узкая, чем в кристалле $\mathrm{LiCl}(2.0 \mathrm{eV})$ и образована $3 d$-состояниями меди.

В настоящей работе примесный атом меди замещает атом лития и помещался в позицию с кубической симметрией во всех расчетах в модели РЭЯ.

Для РЭЯ из 16 примитивных ячеек было проведено сравнение результатов расчета с кубической симметрией и при полном снятии симметрии. Результаты такого сравнения представлены в табл. 4.

Из этой таблицы видно, что полное снятие симметрии мало меняет все полученные результаты: постоянную решетки, ширину запрещенной зоны, полную энергию на ячейку и расстояние $R_{\mathrm{Cu}-\mathrm{Cl}}$. Малым оказывается и расщепление вырожденных примесных $3 d$-уровней меди с симметрией $t_{2 g}$ и $e_{g}$.

В модели РЭЯ, в отличие от модели циклического кластера, рассматривается периодический дефект в кристалле, а выбор РЭЯ определяет не только концентрацию дефекта, но и ширину зоны примесных состояний, которая с увеличением размеров РЭЯ уменьшается до 0 , что соответствует одиночному дефекту в кристалле. 
Таблица 4. Результаты расчета суперъячейки $\mathrm{Li}_{15} \mathrm{CuCl}_{16}$

\begin{tabular}{c|c|c|c}
\hline Свойство & $O_{h}$ & $C_{1}$ & $|\Delta|$ \\
\hline$a(\AA)$ & 8.867 & 8.855 & 0.012 \\
$E_{g}(\mathrm{eV})$ & 5.98 & 5.99 & 0.01 \\
$E$ (a.u.) & -9115.6822 & -9115.6824 & 0.0002 \\
$R_{\mathrm{Cu}-\mathrm{Cl}(\AA)}$ & 2.560 & 2.566 & 0.006 \\
$t_{2 g}$ (a.u.) & -0.22872 & $-0.22935(2),-0.22936$ & $0.0063,0.0064$ \\
$e_{g}$ (a.u.) & -0.20538 & $-0.20663,-0.20658$ & $0.00125,0.00120$
\end{tabular}

Таблица 5. Ширина примесной зоны $E_{w}$ и энергия расщепления между одноэлектронными уровнями $t_{2 g}$ и $e_{g}$ для разных РЭЯ. Все результаты приведены в $\mathrm{eV}$

\begin{tabular}{c|c|c|c|c}
\hline Энергия & $N=4$ & $N=8$ & $N=16$ & $N=32$ \\
\hline$E_{w}\left(t_{2 g}\right)$ & 0.46 & 0.08 & 0.01 & 0.00 \\
$E_{w}\left(e_{g}\right)$ & 1.55 & 0.35 & 0.05 & 0.05 \\
$E\left(e_{g}\right)-E\left(t_{2 g}\right)$ & - & 0.78 & 0.64 & 0.59
\end{tabular}

На рис. 2 показано изменение зоны примесных уровней меди в кристалле $\mathrm{LiCl}$ c ростом элементарной ячейки. Выбраны РЭЯ, преобразующие гцк-решетку в простую кубическую $(F \rightarrow P, N=4)$, в гцк-решетку из РЭЯ $(F \rightarrow F, N=8)$, гцк-решетку в оцк $(F \rightarrow I$, $N=16)$. Матрицы преобразований приведены в табл. 2.

Из рис. 2 видно, что увеличение РЭЯ уменьшает ширину примесной зоны меди. При $N=8$ примесная зона расщепляется на две подзоны, переходящие при $N=16$ в уровни $t_{2 g}$ и $e_{g}$, соответствующие расщеп- лению $d$-уровней меди в кубическом поле. В табл. 5 приведена ширина каждой из примесных подзон, а также величина расщепления между уровнями $t_{2 g}$ и $e_{g}$. Из табл. 5 видно, что величина этого расщепления при $N=32$ (концентрации примеси $3.125 \%$ ) составляет $0.59 \mathrm{eV}$ при экспериментальном значении $0.46 \mathrm{eV}$ [23] для более низкой концентрации примеси, которую можно получить дальнейшим увеличением РЭЯ. В расчетах на основе модели молекулярного кластера (модель одиночного дефекта) [23,24] это расщепление получается равным $0.6 \mathrm{eV}$. Отметим, что при последовательном применении зонной модели дефекта классификация состояний валентной зоны должна проводиться на основе симметрии кристалла с примесью в модели РЭЯ, как это показано на рис. 2. Для РЭЯ из 4, 8 и 16 примитивных ячеек получаются пространственные группы с симметрией $P m \overline{3} m, F m \overline{3} m$ и $\operatorname{Im} \overline{3} m$ соответственно.

Как мы видели из табл. 4, для примесной меди в кристалле $\mathrm{LiCl}$ результаты мало зависят от симметрии, которую замещаемый атом может иметь при расщеплении его позиции Уайкова с введением РЭЯ. Это
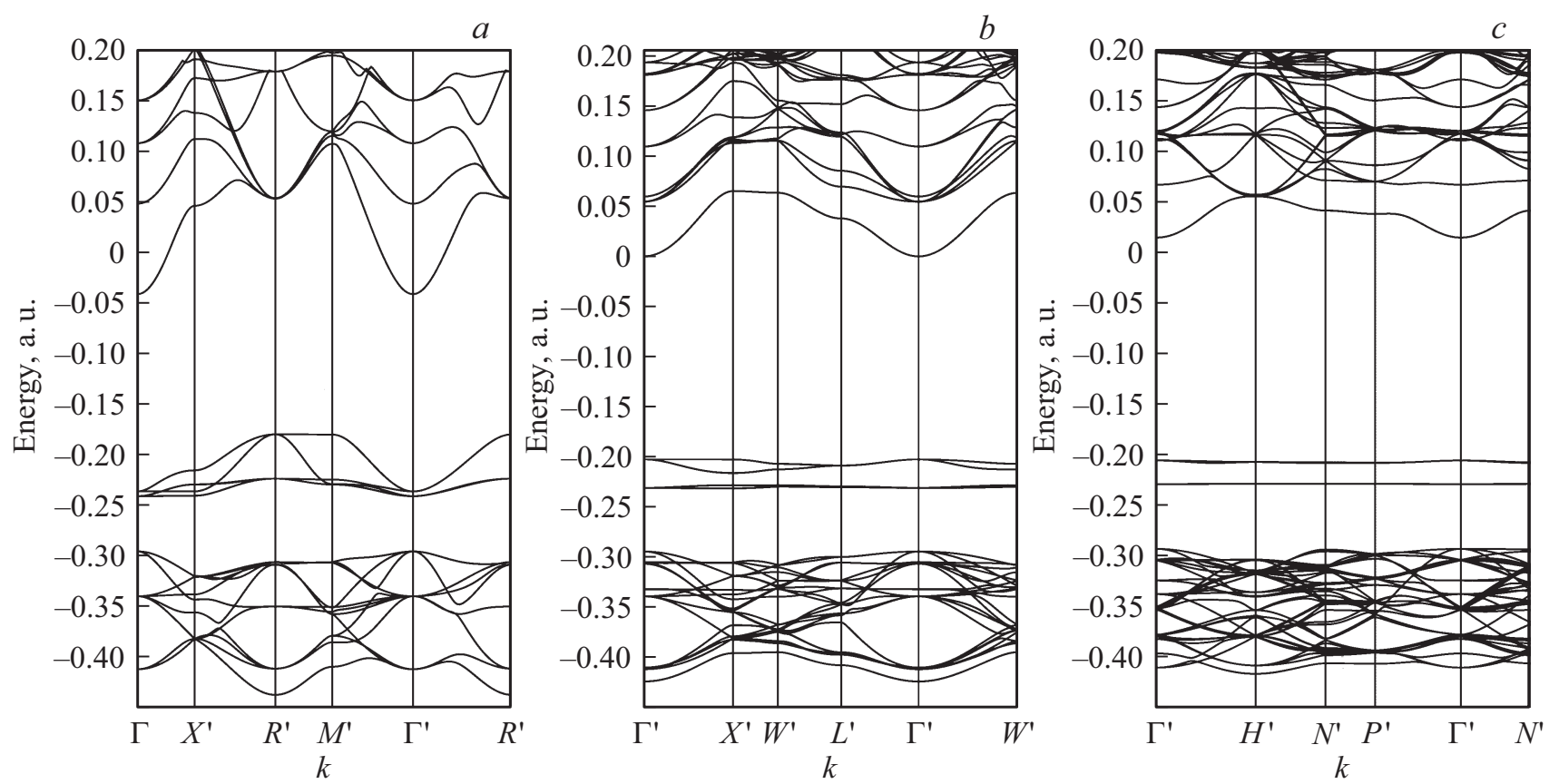

Рис. 2. Зонная модель центра $\mathrm{LiCl}$ : $\mathrm{Cu}$ : рисунки $a-c$ соответствуют РЭЯ из 4,8 и 16 примитивных ячеек соответственно. 
обусловлено как одинаковой симметрией кристаллов $\mathrm{LiCl}$ и $\mathrm{CuCl}$, так и тем, что $3 d$-состояния примеси хорошо локализованы на атоме меди и достаточно далеко отстоят от уровней валентной $3 p$-зоны хлора в кристалле $\mathrm{CuCl}$. Ситуация оказывается иной для центра $\mathrm{SrTiO}_{3}: \mathrm{Fe}$, поскольку симметрия кристаллов $\mathrm{SrTiO}_{3}$ и $\mathrm{SrFeO}_{3}$ является различной.

\section{5. Примесь с понижением локальной симметрии замещаемого атома: $\mathrm{SrTiO}_{3}: \mathrm{Fe}$}

Для примесного атома $\mathrm{Fe}$ в кристалле $\mathrm{SrTiO}_{3}$ проведено достаточно много расчетов [16,25-31]. В большинстве этих расчетов сохраняется кубическая симметрия дефектного кристалла, в то время как экспериментальные данные свидетельствуют о тетрагональной симметрии.

На основе расчетов [25-27] с оптимизацией геометрии был сделан вывод, что эффект Яна-Теллера приводит к тетрагональному искажению, а точечная симметрия примесного центра $\mathrm{SrTiO}_{3}: \mathrm{Fe}-D_{4 h}$.

Учет расщепления в модели РЭЯ занятых атомом Ті позиций в структуре перовскита с группой симметрии $P m \overline{3} m$ позволяет показать, что энергетически наиболее выгодное расположение дефекта соответствует тетрагональной симметрии.

Для структуры перовскита возможны 2 эквивалентных описания со следующим распределением атомов по позициям Уайкова: $\mathrm{Sr} 1 b(0.5,0.5,0.5)$, Ti $1 a(0,0,0)$, $\mathrm{O} 3 d(0.5,0,0)$ и $\mathrm{Sr} 1 a(0,0,0)$, Ti $1 b(0.5,0.5,0.5)$, О $3 c(0.5,0.5,0)$. В обоих случаях атом Ті занимает позицию с локальной симметрией $O_{h}$ - точечной группой из 48 операций симметрии $S 48$.

Пусть в модели РЭЯ используется следующая матрица преобразования от простой кубической к гранецентрированной кубической решетке

$$
L(P \rightarrow F)=\left(\begin{array}{lll}
0 & 2 & 2 \\
2 & 0 & 2 \\
2 & 2 & 0
\end{array}\right)
$$

Определитель матрицы (7) равен 16, то есть к каждой точке суженной зоны Бриллюэна приводятся 15 точек исходной, а каждая позиция Уайкова в примитивной ячейке прямой решетки расщепляется на 16 позиций в РЭЯ новой решетки. Для двух возможных позиций атома Ті в структуре перовскита это расщепление имеет вид (см. [8]):

$$
\begin{gathered}
a(0,0,0)=a^{(16)}(0,0,0)+b^{(16)}(0.5,0.5,0.5) \\
+2 c^{(16)}(0.25,0.25,0.25) \\
+6 d^{(16)}(0,0.25,0.25)+6 e^{(16)}(0.25,0,0) \\
b(0.5,0.5,0.5)=8 f^{(16)}(0.125,0.125,0.125) \\
+8 f^{(16)}(0.375,0.375,-0.125) .
\end{gathered}
$$

В левой части равенств (8) координаты позиций Уайкова заданы для исходной (построенной из примитивных яче-

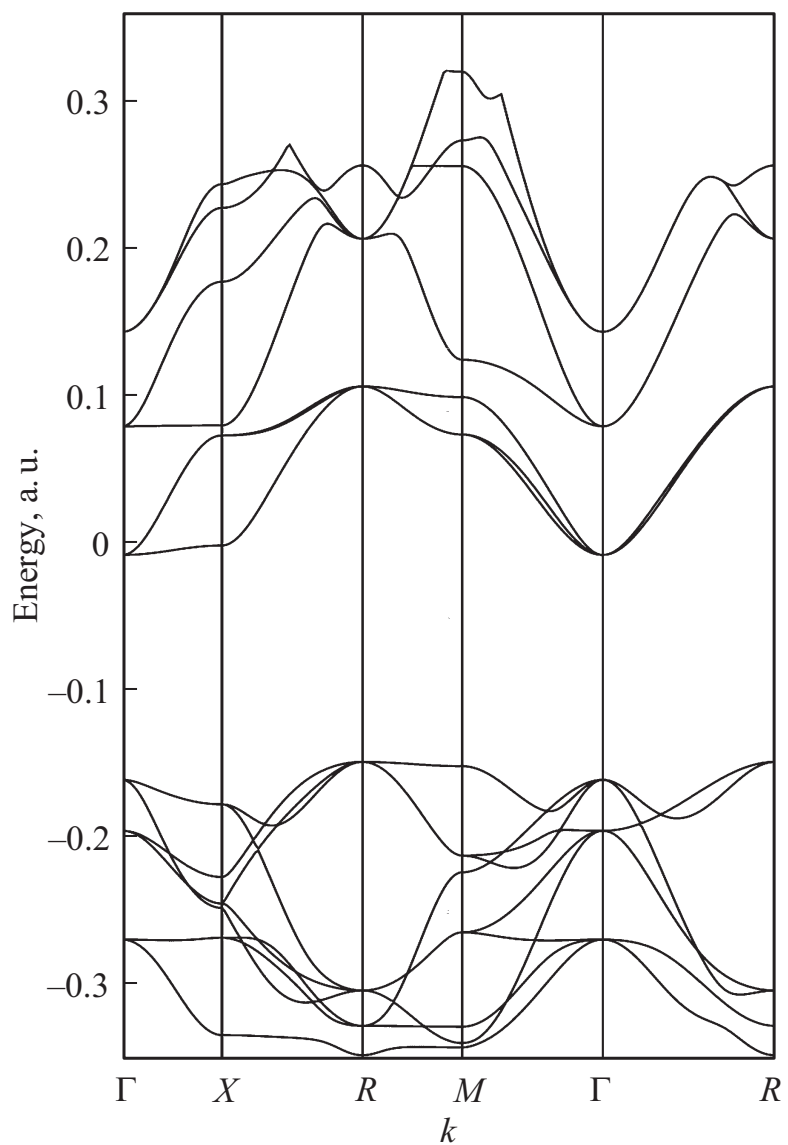

Pис. 3. Зонная структура кристалла $\mathrm{SrTiO}_{3}$.

ек) пространственной группы $P m \overline{3} m$, а в правой - для построенной из РЭЯ пространственной группы $F m \overline{3} m$.

В табл. 6 приведено расщепление $d$-орбиталей в полях с точечной симметрией позиций Уайкова из (8). $\mathrm{Sn}$ означает число операций симметрии в точечной группе. Добавлена также точечная группа $D_{4 h}$, с которой связывают реальную симметрию примесного железа в титанате стронция.

Из этой таблицы видно, что для свободного иона $\mathrm{Fe}^{4+}$ с электронной конфигурацией валентных электронов $3 d^{4}$ и расщеплением $t_{2 g}^{3} e_{g}^{1}$ в кубическом или тетраэдрическом поле эффект Яна-Теллера должен привести к снятию вырождения и понижению симметрии как минимум до тетрагональной с конфигурацией $b_{2 g}^{1} e_{g}^{2} a_{1 g}^{1} b_{1 g}$. Этот вывод согласуется и с тетрагональной симметрией кристалла $\mathrm{SrFeO}_{3}$.

В табл. 7 проведено сравнение симметрии кристаллов $\mathrm{SrTiO}_{3}$ и $\mathrm{SrFeO}_{3}$. Из табл. 7 видно, что при полном замещении атомов Ti на атом Fe кубическая симметрия понижается до тетрагональной. Такое понижение свойственно и примеси Fe в узле Ti.

Выше отмечалось, что при выборе РЭЯ необходимо учитывать симметрию одноэлектронных состояний для вершины валентной зоны и дна зоны проводимости. На рис. 3 показана рассчитанная нами методом DFT PBE0 зонная структура кристалла $\mathrm{SrTiO}_{3}$. Из рис. 3 сле- 
Таблица 6. Расщепление $d$-орбиталей в полях с различной точечной симметрией

\begin{tabular}{c|c|c|c|c|c}
\hline $\begin{array}{c}O_{h}(S 48) \\
1 a(0,0,0)\end{array}$ & $\begin{array}{c}T_{d}(S 24) \\
2 c(0.25,0.25,0.25)\end{array}$ & $D_{4 h}(S 16)$ & $\begin{array}{c}C_{4 v}(S 8) \\
6 e(0.25,0,0)\end{array}$ & $\begin{array}{c}C_{2 h}(S 4) \\
6 d(0,0.25,0.25)\end{array}$ & $\begin{array}{c}C_{3 v}(S 6) \\
8 f(0.125,0.125,0.125)\end{array}$ \\
\hline$e_{g}$ & $E$ & $a_{1 g}, b_{1 g}$ & $a_{1}, b_{1}$ & $a_{g}, a_{g}$ & $a_{1}, e$ \\
$\left(x^{2}-y^{2}, z^{2}\right)$ & $\left(x^{2}-y^{2}, z^{2}\right)$ & $z^{2}, x^{2}-y^{2}$ & $z^{2}, x^{2}-y 2$ & $z^{2}-y^{2}$ & $z^{2},\left(x^{2}-y^{2}, x y\right)$ \\
\hline$t_{2 g}$ & $T$ & $b_{2 g}, e_{g}$ & $b_{2}, e$ & $b_{1 g}, b_{2 g}, b_{3 g}$ & $e$ \\
$(x y, x z, y z)$ & $(x y, x z, y z)$ & $x y,(x z, y z)$ & $x y,(x z, y z)$ & $x y, x z, y z$ & $(x z, y z)$
\end{tabular}

Таблица 7. Симметрия кристаллов $\mathrm{SrTiO}_{3}$ и $\mathrm{SrFeO}_{3}$

\begin{tabular}{|c|c|c|c|c|c|}
\hline \multicolumn{3}{|c|}{$\mathrm{SrTiO}_{3}\left(O_{h}^{1}-221\right)$} & \multicolumn{3}{|c|}{$\mathrm{SrFeO}_{3}\left(C_{4 v}^{1}-99\right)$} \\
\hline $\mathrm{Sr}$ & $O_{h}$ & $1 b(0.5,0.5,0.5)$ & $\mathrm{Sr}$ & $C_{4 v}$ & $1 b(0.5,0.5,0.524)$ \\
\hline $\mathrm{Ti}$ & $O_{h}$ & $1 a(0,0,0)$ & $\mathrm{Fe}$ & $C_{4 v}$ & $1 a(0,0,0)$ \\
\hline $\mathrm{O}$ & $D_{4 h}$ & $3 d(0.5,0,0 ; 0,0.5,0 ; 0,0,0.5)$ & $\begin{array}{l}\mathrm{O}_{1} \\
\mathrm{O}_{2}\end{array}$ & $\begin{array}{l}C_{2 v} \\
C_{4 v}\end{array}$ & $\begin{array}{c}2 c(0.5,0,0.0329) \\
1 a(0,0,0.5465)\end{array}$ \\
\hline
\end{tabular}

Таблица 8. Расчет РЭЯ $\mathrm{Sr}_{16} \mathrm{Ti}_{15} \mathrm{FeO}_{48}$ с симметрией пространственной группы $F m \overline{3} m$. $(\Delta E-$ изменение энергии в результате полной оптимизации геометрии кристалла с дефектом; $E_{f}, E_{f o p t}-$ энергия образования дефекта до и после оптимизации геометрии. Все результаты приведены в $\mathrm{eV}$

\begin{tabular}{c|c|c|c|c|c|c|c}
\hline Энергия & $\begin{array}{c}O_{h}(S 48) \\
1 a(0,0,0)\end{array}$ & $\begin{array}{c}T_{d}(S 24) \\
2 c(0.25,0.25,0.25)\end{array}$ & $D_{4 h}(S 16)$ & $\begin{array}{c}C_{4 v}(S 8) \\
6 e(x, 0,0)\end{array}$ & $\begin{array}{c}C_{2 h}(S 4) \\
6 d(0,0.25,0.25)\end{array}$ & $\begin{array}{c}C_{3 v}(S 6) \\
8 f(x, x, x)\end{array}$ & $\begin{array}{c}C_{1}(S 1) \\
48 l(x, y, z)\end{array}$ \\
\hline$\Delta E$ & 2.46 & -0.10 & -0.46 & -0.44 & -0.38 & -0.73 & -0.33 \\
$E_{f}$ & 8.81 & 8.81 & 6.43 & 6.43 & 6.43 & 8.01 & 6.43 \\
$E_{f \text { opt }}$ & 11.27 & 8.72 & 5.97 & 5.99 & 6.05 & 7.28 & 6.09
\end{tabular}

дует, что в кристалле $\mathrm{SrTiO}_{3}$ щель не прямая: вершине валентной зоны соответствует точка $R(0.5,0.5,0.5)$ зоны Бриллюэна для простой кубической решетки, а дну зоны проводимости - точка $\Gamma(0,0,0)$.

Соотношение (2) для выбранной РЭЯ приводит к следующему преобразованию точек симметрии в обратной решетке

$$
\begin{aligned}
& \Gamma^{\prime}(0,0,0): \Gamma(0,0,0)+R(1 / 2,1 / 2,1 / 2)+3 X(1 / 2,0,0) \\
&+3 M(1 / 2,1 / 2,0)+8(1 / 4,1 / 4,-1 / 4), \\
& 3 X^{\prime}(1 / 2,0,1 / 2): 6(1 / 4,0,0)+6(1 / 2,1 / 2,1 / 4) \\
&+12(1 / 2,-1 / 4,1 / 4)+12(0,1 / 4,-1 / 4) \\
&+12(1 / 2,-1 / 4,0), \\
& 4 L^{\prime}(1 / 2,1 / 2,1 / 2): 8(1 / 8,1 / 8,1 / 8)+8(3 / 8,3 / 8,-3 / 8) \\
&+24(0,3 / 8,1 / 4)+24(1 / 8,3 / 8,-1 / 8),
\end{aligned}
$$

$6 W^{\prime}(1 / 2,1 / 4,3 / 4): 24(1 / 4,1 / 8,0)+24(-1 / 4,1 / 2,1 / 8)$

$$
+24(1 / 4,3 / 8,0)+24(1 / 2,-1 / 4,3 / 8)
$$

Из соотношения (9а) следует, что трансляционная симметрия границ запрещенной зоны исходного кристалла (точки $\Gamma$ и $R$ ) воспроизводится для выбранной РЭЯ (80 атомов), как для модели периодического дефекта, так и для циклического кластера, моделирующего одиночный дефект в кристалле.

В табл. 8 приведены результаты расчета полной энергии для модели РЭЯ при различном выборе позиции Уайкова для примесного атома Fе. Расчеты проведены с учетом спиновой поляризации и для максимальной проекции спина на примеси железа $S_{z}=4$. Приводится изменение полной энергии на ячейку в результате полной оптимизации геометрии кристалла с дефектом и энергия образования дефекта до $\left(E_{f}\right)$ и после $\left(E_{f \text { opt }}\right)$ оптимизации, рассчитанные с помощью соотношения

$$
E_{f}=E_{\text {perf }}-E_{\mathrm{Ti}}+E_{\mathrm{Fe}}-E_{\text {def. }} .
$$

В (10) $E_{\text {perf }}-$ умноженная на 16 энергия на примитивную ячейку исходного кристалла $\mathrm{SrTiO}_{3}, E_{\mathrm{Ti}}, E_{\mathrm{Fe}}-$ энергии на атом, рассчитанные для кристаллических титана и железа (с симметрией пространственной группы $P 6_{3} / m m c$ и $\operatorname{Im} \overline{3} m$ соответственно); $E_{\text {def. }}$ 一энергия кристалла с примесью в модели РЭЯ.

Из табл. 8 следует, что энергия образования примесного железа в титанате стронция существенно зависит от симметрии узла, где находится замещаемый 
атом титана в РЭЯ. Основному состоянию примеси соответствует энергия образования около $6 \mathrm{eV}$, а симметрия при этом - тетрагональная. Численное значение полученной нами энергии образования хорошо согласуется с результатами других расчетов в модели РЭЯ: $6.4 \mathrm{eV} \mathrm{[16],} 6.1 \mathrm{eV}$ [30] для 80 и 100 атомов соответственно. Для РЭЯ из 180 атомов энергия образования $E_{f}$ уменьшается до $4.7 \mathrm{eV}$ [31], что, по-видимому, связано с увеличением периода дефекта и как следствие, ослаблением взаимодействия между дефектами в зонной модели.

Наряду с рассмотренными выше дефектами замещения, в кристаллах могут существовать и междоузельные атомы, взаимодействующие с атомами кристалламатрицы. Для описания таких дефектов приходится учитывать расщепление позиций Уайкова, которые в исходном кристалле вакантны. В следующем параграфе рассчитан дефект такого рода $-H$ центр в кристалле $\mathrm{CsPbI}_{3}$.

\section{6. Примесный атом йода в междоузлии (Н-центр) в кристалле $\mathrm{CsPbl}_{3}$}

Как и в случае рассмотренных выше дефектов, в начале рассчитываются свойства кристалла $\mathrm{CsPbI}_{3}$ без дефекта. Симметрия этого кристалла определяется пространственной группой $P m \overline{3} m$ с тремя занятыми позициями Уайкова $1 a \mathrm{~Pb}(0,0,0), 1 b \mathrm{Cs}(0.5,0.5,0.5), 3 d$ I $(0.5,0,0)$, не содержащими свободных параметров. Рассчитанное методом PBESOL0 с оптимизацией геометрии значение постоянной решетки $6.33 \AA$ близко к экспериментальному значению $6.29 \AA$. Малликеновские заряды $+0.99,+0.85,-0.61$ на атомах $\mathrm{Cs}, \mathrm{Pb}$ и I соответственно подтверждают сделанный ранее на основе других расчетов вывод о том, что взаимодействие атомов йода и свинца является существенно ковалентным. Практически равный +1 заряд на атоме Cs равен заряду метиламмоний группы $\left(\mathrm{CH}_{3}-\mathrm{NH}_{3}\right)^{+}$в гибридных галоидных перовскитах [32,33].

Зонная структура кристалла $\mathrm{CsPbI}_{3}$, рассчитанная нами с гибридным функционалом плотности PBESOL0, представлена на рис. 4. В согласии с данными других расчетов [34], энергетическая щель прямая (в точке $R$ зоны Бриллюэна), верхняя валентная зона образована $5 p$-состояниями атома йода с небольшой примесью $6 s$-состояний свинца, а дно зоны проводимости определяется 6р-орбиталями атома свинца. Наше расчетное значение ширины запрещенной зоны $2.7 \mathrm{eV}$ завышено по сравнению с экспериментальным значением $1.7 \mathrm{eV}$. Расчеты [34] приводят к уменьшению ширины запрещенной зоны на $1.1 \mathrm{eV}$ при учете спинорбитального взаимодействия. Этот учет важен, прежде всего, для положения дна зоны проводимости и мало влияет на положение вершины валентной зоны. Таким образом, поправка рассчитанной нами ширины запрещенной зоны на спин-орбитальное взаимо-

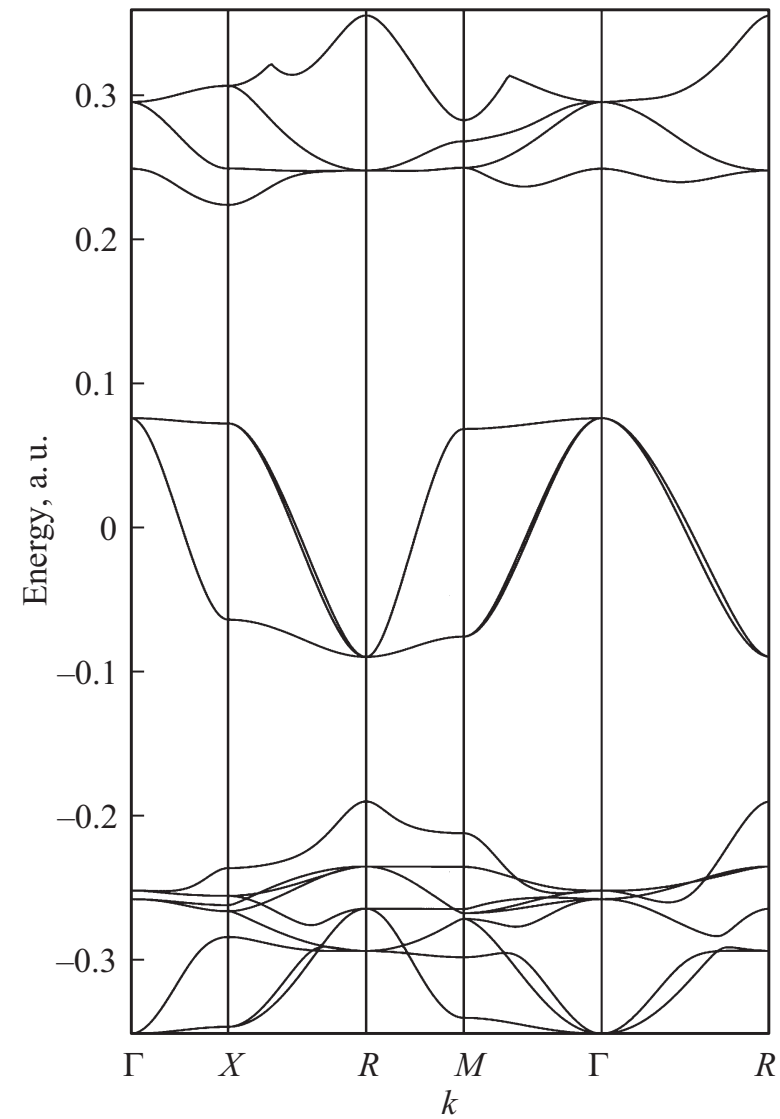

Рис. 4. Зонная структура кристалла $\mathrm{CsPbI}_{3}$.

действие приводит к хорошему согласию с экспериментом. Поскольку поправка для вершины валентной зоны мала, положение уровней $H$-центра в запрещенной зоне будем отсчитывать от вершины валентной зоны.

$H$-центр в кристалле $\mathrm{CsPbI}_{3}$ - это примесный нейтральный атом йода в междоузлии, создающий с ионом йода основного кристалла молекулярный ион $\mathrm{I}_{2}^{-}$[35]. Мы рассчитали этот дефект в модели РЭЯ $2 \times 2 \times 2$, т.е. с удвоением каждого из векторов трансляции исходной простой кубической решетки. При этом период одиночного дефекта равен вектору трансляции РЭЯ $12.66 \AA$, то есть достаточно велик. Существенно, что такой выбор РЭЯ позволяет воспроизвести энергию исходного кристалла в вершине валентной зоны (точке $R$ зоны Бриллюэна).

Поместим нейтральный атом йода в вакантную в исходном кристалле позицию $3 c$, которая для рассматриваемой РЭЯ расщепляется на две позиции с одинаковой точечной симметрией $C_{2 v}: 12 i(0,0.25,0.25)$, $12 j(0.5,0.25,0.25)$.

В табл. 9 представлены результаты расчетов $H$-центра в кристалле $\mathrm{CsPbI}_{3}$, полученные как с учетом $C_{2 v}$ симметрии, так и без такого учета. В обоих случаях проводилась полная оптимизация геометрии. При этом результаты практически совпадают для примеси в позициях $12 i$ или $12 j$. 
Таблица 9. Результаты расчета $H$-центра в кристалле $\mathrm{CsPb}_{3}$

\begin{tabular}{c|c|c|c}
\hline Свойство & $C_{2 v}$ & $C_{1}$ & $\Delta^{\dagger}$ \\
\hline$a, b, c(\AA)$ & $12.66,12.66,12.76$ & $12.63,12.70,12.60$ & $0.03,0.03,0.16$ \\
$\alpha, \beta, \gamma(\mathrm{deg})$ & $90.00,90.00,87.87$ & $90.09,89.76,89.01$ & $0.09,0.24,0.99$ \\
$E_{0}$ & -474.023911 a.u. & -474.041812 a.u. & $0.49 \mathrm{eV}$ \\
$E_{1}$ & -474.152764 a.u. & -474.175555 a.u. & $0.62 \mathrm{eV}$ \\
$\left(E_{0}-E_{1}\right)(\mathrm{eV})$ & 3.51 & 3.64 & 0.13 \\
$E_{\text {def. }}(\mathrm{eV})$ & 0.56 & 0.94 & 0.38 \\
$E_{f}(\mathrm{eV})$ & -0.25 & 0.37 & $0.52,0.01$ \\
$R_{\mathrm{I}-\mathrm{I}}(\AA)$ & $3.84,3.84$ & $3.32,3.83$ & -0.04 \\
$q_{H}(e)$ & -0.39 & -0.35 & -0.13 \\
$q_{1}(e)$ & -0.55 & -0.42 & 0.06
\end{tabular}

Примечание. ${ }^{\dagger} \Delta-$ разность значений свойств, полученных с учетом $C_{2 v}$ симметрии и без такого учета.

В табл. $9 a, b, c, \alpha, \beta, \gamma$ - длины векторов трансляции РЭЯ и значения углов между ними; $E_{0}, E_{1}-$ энергии до и после оптимизации геометрии, $E_{\mathrm{def}}$ и $E_{f}-$ энергия уровня дефекта относительно вершины валентной зоны и энергия образования дефекта; $R_{\mathrm{I}-\mathrm{I}}-$ расстояние от примесного атома йода до двух атомов йода кристалла, $q$ - малликеновские заряды на соответствующих атомах.

Из приведенных в табл. 9 результатов видно следующее:

а) учет релаксации кристалла с дефектом (оптимизация геометрии) заметно понижает полную энергию на ячейку, однако это понижение мало зависит от выбранной точечной симметрии;

б) оптимизированные вектора трансляции РЭЯ и углы между ними мало отличаются от исходных величин $12.66 \AA$ и $90 \AA$, но при снятии симметрии это отличие несколько увеличивается;

в) симметрия выбранной модели существенно влияет на рассчитанное положение уровня дефекта относительно вершины валентной зоны исходного кристалла (при снятии симметрии оно увеличивается на $0.38 \mathrm{eV}$ );

г) распределение зарядов на атомах также зависит от симметрии модели: для симметрии $C_{2 v}$ заряды на двух атомах йода кристалла одинаковы $(-0.55 e)$ и близки к заряду на атомах йода в исходном кристалле $(-0.62 e)$; для симметрии $C_{1}$ заряды на ближайшем к примеси атоме йода и на самой примеси изменяются (суммарный заряд на этих двух атомах равен $-0.77 e$ и соответствует иону $\mathrm{I}_{2}^{-}$в кристалле), а заряд на втором соседе примеси практически совпадает с зарядом на атоме в исходном кристалле;

д) молекулярный ион $\mathrm{I}_{2}^{-}$появляется только при полном снятии симметрии: расстояние от межузельного атома йода до ближайшего атома йода исходного кристалла уменьшается от $3.84 \AA$ (до оптимизации геометрии это расстояние равно $4.48 \AA$ ) до $3.32 \AA$ и практически совпадает с межатомным расстоянием $3.35 \AA$ в свободной молекуле $\mathrm{I}_{2}^{-}$. Последнее рассчитано нами с тем же функционалом PBESOLO и тем же атомным базисом, что использовались в расчете кристалла $\mathrm{CsPb}_{3}$.
Энергия $E_{f}$ образования иона $\mathrm{I}_{2}^{-}$в кристалле получена с помощью соотношения:

$$
E_{f}=E_{\text {perf }}+E\left(\mathrm{I}_{2}^{-}\right) / 2-E_{\text {def. }} .
$$

В (11) $E_{\text {perf }}-$ умноженная на 8 энергия на примитивную ячейку исходного кристалла, $E\left(\mathrm{I}_{2}^{-}\right)$- энергия основного состояния молекулы $\mathrm{I}_{2}^{-}, E_{\mathrm{def}}-$ энергия кристалла с примесью в модели РЭЯ. Вычисленная по (11) энергия образования молекулярного иона $\mathrm{I}_{2}^{-}$равна $0.37 \mathrm{eV}$, а при сохранении симметрии $C_{2 v}$ энергия $E_{\text {def. }}$ оказывается выше, чем $\left(E_{\mathrm{perf}}+E\left(\mathrm{I}_{2}^{-}\right) / 2\right)$, то есть энергия образования, вычисленная по (11), отрицательна. Это показывает, что $H$-центр в кристалле $\mathrm{CsPb}_{3}$ имеет симметрию $C_{1}$.

\section{7. Выводы}

Основные выводы из настоящей работы сводятся к следующему.

1. При рассмотрении точечного дефекта в кристалле в модели расширенной элементарной ячейки необходимо учитывать симметрию одноэлектронных состояний исходного кристалла в вершине валентной зоны и на дне зоны проводимости. Выбранная РЭЯ должна воспроизводить эти состояния.

2. Для правильного определения расположения примесных уровней дефекта в запрещенной зоне исходного кристалла электронная структура последнего должна рассчитываться с учетом изменения классификации состояний по волновому вектору для каждой конкретной РЭЯ.

3. Каждому конкретному выбору РЭЯ соответствует расщепление позиций Уайкова исходного кристалла и как результат - возможность поместить дефект в позиции с разной точечной симметрией, а также провести расчет без учета точечной симметрии кристалла с дефектом вообще. Такой подход к модели РЭЯ известен как метод сайт симметрии.

4. По результатам расчета кристалла с дефектом в модели РЭЯ с учетом сайт-симметрии можно определить реальную симметрию кристалла с дефектом, 
что существенно для интерпретации экспериментальных данных.

5. Показано, что примесь меди в узле лития в кристалле $\mathrm{LiCl}$ сохраняет кубическую симметрию замещаемого атома, примесь атома железа в узле титана с кубической локальной симметрией в кристалле $\mathrm{SrTiO}_{3}$ понижает симметрию до тетрагональной, а для $H$ центра в кристалле $\mathrm{CsPbI}_{3}$ с образованием иона $\mathrm{I}_{2}^{-}$характерно полное снятие точечной симметрии.

\section{Список литературы}

[1] C. Freysoldt, B. Grabowski, T. Hickel, J. Neugebauer, G. Kresse. Rev. Mod. Phys. 86, 253 (2014).

[2] R.A. Evarestov. Phys. Status Solidi A 202, 235 (2005).

[3] P. Deak. Phys. Status Solidi B 217, 9 (2000).

[4] R.A. Evarestov, A.V. Bandura. Theor. Chem. Acc. 137, 14 (2018).

[5] R.A. Evarestov, Yu.E. Kitaev, V.V. Porsev. J. Appl. Cryst. 50, 893 (2017).

[6] R. Dovesi, V.R. Saunders, C. Roetti, R. Orlando, C.M. Zicovich-Wilson, F. Pascale, B. Civalleri, K. Doll, N.M. Harrison, I.J. Bush, Ph. D’Arco, M. Llunell, M. Causá, Y. Noel. CRYSTAL17 User's Manual. University of Turin, Torino (2017).

[7] G. Kresse, M. Marsman, J. Furthmuller. VASP the GUIDE, http://cms.mpi.univie.ac/vasp

[8] Bilbao Crystallographic Server. http://www.cryst.ehu.es. Accessed 01 DEC 2018

[9] R.A. Evarestov, S. Piskunov, Yu.F. Zhukovskii. Chem. Phys. Lett. 682, 91 (2017).

[10] R.A. Evarestov, A. Platonenko, D. Gryaznov, Yu.F. Zhukovskii, E.A. Kotomin. Phys. Chem. Chem. Phys. 19, 25245 (2017).

[11] R.A. Evarestov, A. Platonenko, Yu.F. Zhukovskii. Comput. Mater. Sci. 150, 517 (2018).

[12] R.A. Evarestov, D. Gryaznov, M. Arrigoni, E.A. Kotomin, A. Chesnokov. J. Maier. Phys. Chem. Chem. Phys. 19, 8340 (2017).

[13] R.A. Evarestov. Quantum Chemistry of Solids. The LCAO First Principles Treatment of Crystals and Nanostructures. Springer Series in Solid State Sciences $153,2^{\text {nd }}$ ed. SpringerVerlag, Berlin-Heidelberg (2012). 734 p.

[14] R.A. Evarestov, M.I. Petrashen, E.M. Ledovskaya. Phys. Status Solidi B 76, 377 (1976).

[15] T. Bredow, R.A. Evarestov, K. Jug. Phys. Status Solidi B 222, 495 (2000).

[16] Z. Chao, W. Chun-Lei, L. Ji-Chao, Y. Kun. Chinese Phys. 16, 1422 (2007).

[17] Yu.E. Kitaev, P. Tronc. Phys. Solid State 54, 520 (2012).

[18] C. Adamo, V. Barone. J. Chem. Phys. 110, 6158 (1999).

[19] J.P. Perdew, A. Ruzsinszky, G.I. Csonka, O.A. Vydrov, G.E. Scuseria, L.A. Constantin, X. Zhou, K. Burke. Phys. Rev. Lett. 100, 136406 (2008).

[20] Crystal Home Page http://www.crystal.unito.it Basis Sets. Accessed 01 Dec 2018.

[21] R.A. Evarestov, E. Blokhin, D. Gryaznov, E.A. Kotomin, J. Maier. Phys. Rev. B 83, 134108 (2011).

[22] H.J. Monkhorst, J.D. Pack. Phys. Rev. B 13, 5188 (1976).

[23] J.G. Harrison, C.C. Lin. Phys. Rev. B 23, 3894 (1981).

[24] R.A. Heaton, J.G. Harrison, C.C. Lin. Phys. Rev. B 31, 1077 (1985).
[25] R.A. Evarestov, S. Piskunov, E.A. Kotomin, G. Borstel. Phys. Rev. B 67, 064101 (2003).

[26] V.E. Alexandrov, J. Maier, R.A. Evarestov. Phys. Rev. B 77, 075111 (2008).

[27] S. Piskunov. Computer Modelling \& New Technologies 6, 29 (2002).

[28] E. Blokhin, E.A. Kotomin, J. Maier. J. Phys. Condens. Matter 24, 104024 (2012)

[29] E. Blokhin, E. Kotomin, A. Kuzmin, J. Purans, R. Evarestov, J. Maier. Appl. Phys. Lett. 102, 112913 (2013).

[30] X. Zhou, J. Shi, C. Li. J. Phys. Chem. C. 115, 8305 (2011).

[31] J.N. Baker, P.C. Bowes, D.M. Long, A. Moballegh, J.S. Harris, E.C. Dickey, D.L. Irving. Appl. Phys. Lett. 110, 122903 (2017).

[32] C. Eames, J.M. Frost, P.R.F. Barnes, B.C. O’Regan, A. Walsh, M.S. Islam. Nature Commun. 6, 7497 (2015).

[33] M.E. Madjet, F. El-Mellouhi, M.A. Carignano, G.R. Berdiyorov. J. Appl. Phys. 119, 165501 (2016).

[34] L. Lang, J.-H. Yang, H.-R. Liu, H.J. Xiang, X.G. Gong. Phys. Lett. A 378, 290 (2014).

[35] L.D. Whalley, R. Crespo-Otero, A. Walsh. ACS Energy Lett. 2, 2713 (2017).

Редактор Ю.Э. Китаев 